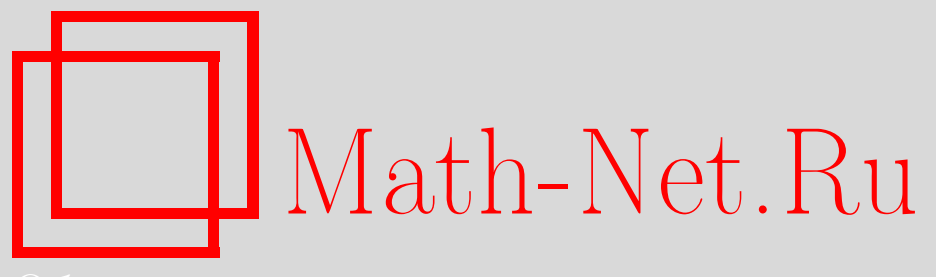

А. А. Новиков, Несколько замечаний о распределении времени первого выхода и оптимальной остановке AR(1)-последовательностей, Теория вероятн. и ее примен., 2008, том 53, выпуск 3, 458-471

DOI: https://doi.org/10.4213/tvp2442

Использование Общероссийского математического портала MathNet.Ru подразумевает, что вы прочитали и согласны с пользовательским соглашением

http: //www . mathnet.ru/rus/agreement

Параметры загрузки:

IP : 54.205 .225 .156

26 апреля 2023 г., 16:54:04

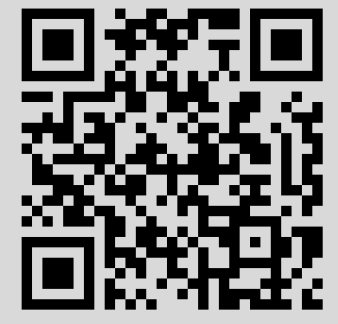




\title{
НЕСКОЛЬКО ЗАМЕЧАНИЙ О РАСПРЕДЕЛЕНИИ ВРЕМЕНИ ПЕРВОГО ВЫХОДА И ОПТИМАЛЬНОЙ ОСТАНОВКЕ AR(1)-ПОСЛЕДОВАТЕЛЬНОСТЕЙ ${ }^{1)}$
}

\begin{abstract}
В работе выводятся достаточные условия для экспоненциальной ограниченности времени выхода за уровень авторегрессионной последовательности первого порядка $(\mathrm{AR}(1))$ и доказывается тождество для среднего времени первого выхода. Далее это тождество используется для вывода логарифмической асимптотики среднего времени первого выхода гауссовской $\mathrm{AR}(1)$-последовательности из полосы. Точность асимптотической аппроксимации иллюстрируется результатами статистического моделирования, и указывается поправочный коэффициент, приводящий к существенно лучшей точности. Для случая $\mathrm{AR}(1)$-последовательности, порожденной инновацией с экспоненциальным распределением, выводится явная формула для производящей функции времени первого пересечения уровня, которая далее используется для исследования задачи об оптимальной остановке.
\end{abstract}

Ключевые слова и фразы: время первого выхода, авторегрессионные процессы, мартингалы, экспоненциальная ограниченность, оптимальная остановка.

1. Введение. $\mathrm{AR}(1)$-последовательность определяется как решение уравнения

$$
X_{n}=\lambda X_{n-1}+\eta_{n}, \quad n=1,2, \ldots, \quad X_{0}=x,
$$

где $\left\{\eta_{n}\right\}$ - последовательность независимых одинаково распределенных случайных величин (инноваиия), $x$ и $\lambda$ - неслучайные константы. Положим

$$
\tau_{a}=\inf \left\{n \geqslant 0: X_{n}>a\right\} \quad \text { и } \quad \tau_{a, b}=\inf \left\{n \geqslant 0: X_{n}>a \text { или } X_{n}<b\right\},
$$

где мы всегда предполагаем, что inf $\{\varnothing\}=\infty$.

Для случая $\lambda=1$, т.е. когда $X_{n}$ является случайным блужданием, в литературе есть много результатов о распределениях $\tau_{a}$ и перескока

* Математический институт им. В. А. Стеклова PAH; University of Technology Sydney, Department of Mathematical Sciences, PO Box 123, Broadway, NSW 2007, Australia; e-mail: Alex.Novikov@uts.edu.au

1) Работа выполнена при поддержке ARC Discovery Grant. 
$X_{\tau_{a}}-a$. Изложение результатов в этом направлении, полученных, в основном, с помощью факторизационной техники Винера-Хопфа, имеется в [4], [11] и многих других монографиях.

Для времени первого выхода $\tau_{a, b}$ известен следующий результат об экспоненциальной ограниченности (см. [24], [26]): если $\lambda=1$, то

$$
\mathbf{P}\left\{\eta_{1}=0\right\}<1 \Longrightarrow \tau_{a, b} \in \mathrm{Cr},
$$

где $\mathrm{Cr}$ обозначает класс неотрицательных случайных величин с конечным экспоненциальным моментом, т.е.

$$
\xi \in \mathrm{Cr} \Longleftrightarrow \mathbf{E} e^{\alpha \xi}<\infty \quad \text { для некоторого } \alpha>0
$$

(условие Крамера).

Отметим, что в случае $\lambda=0$, очевидно,

$$
\mathbf{P}\left\{b \leqslant \eta_{1} \leqslant a\right\}<1 \Longrightarrow \tau_{a, b} \in \mathrm{Cr} .
$$

Некоторые результаты о распределении времени первого выхода $\tau_{a}$ для случая $\lambda \in(0,1)$ можно найти в работах [14], [15], [19]. В частности, в [19] показано, что если

$$
\mathbf{E}\left(\eta_{1}^{-}\right)^{\delta}<\infty \quad \text { для некоторого } \delta>0
$$

(где $\left.x^{-}=\max (-x, 0)\right)$, то при $a>0$

$$
\mathbf{P}\left\{\eta_{1} \leqslant a(1-\lambda)\right\}<1 \Longrightarrow \tau_{a} \in \mathrm{Cr} ;
$$

кроме того, при выполнении условия

$$
\psi(u):=\ln \mathbf{E} e^{u \eta_{1}}<\infty \quad \text { для всех } u \in[0, \infty)
$$

справедливо следующее тождество:

$$
\mathbf{E}_{x} \tau_{a}=\frac{1}{|\ln \lambda|} \int_{0}^{\infty}\left(\mathbf{E}_{x} e^{u X_{\tau_{a}}}-e^{u x}\right) e^{-\phi(u)} \frac{d u}{u},
$$

где $\phi(u)=\sum_{k=0}^{\infty} \psi\left(\lambda^{k} u\right)$ и символ математического ожидания $\mathbf{E}_{x}$ соответствует начальному условию $X_{0}=x$.

В настоящей работе мы приводим ряд обобшений результатов статьи [19]; в частности, для случая $|\lambda|<1$ мы даем аналог импликации (4) (см. теорему 1 в п. 2) и приводим формулу для математического ожидания $\tau_{a, b}$ (см. теорему 2 в п. 2). В качестве простого следствия последнего результата мы выводим нижнюю оценку и асимптотику для $\mathbf{E}_{x} \tau_{a,-a}$ при $a \rightarrow \infty$ для случая гауссовской инновации $\eta_{n}$ с нулевым средним (верхняя оценка для $\mathbf{E}_{x} \tau_{a,-a}$ в этом случае была получена в [10]).

В п. 3 мы обсуждаем случай, когда инновация $\eta_{n}$ имеет экспоненциальное распределение $\mathbf{P}\left\{\eta_{n}>y\right\}=e^{-y}, y>0$, которое, конечно, 
не удовлетворяет предположению (5). Здесь, используя мартингальный подход, мы выводим явные формулы для $\mathbf{E}_{x} \tau_{a}$ и $\mathbf{E}_{x} \rho^{\tau_{a}}, \rho \in(0,1)$ (см. теорему 3), которые были уже приведены (без доказательства) в лекционных заметках [18]. Отметим, что в работе [12], мотивированной физическими приложениями, была найдена явная формула для $\mathbf{E}_{x} \tau_{0}$ для случая, когда инновация $\eta_{n}$ имеет двустороннее экспоненциальное распределение с $\mathbf{P}\left\{\left|\eta_{n}\right|>y\right\}=\frac{1}{2} e^{-y}, y>0$.

Теорему 3 можно использовать для анализа некоторых схем контроля качества, в которых наблюдаемые случайные величины имеют экспоненциальное распределение (см. [2]). Еще одно применение теоремы 3 дается в п. 4, где обсуждается задача об оптимальной остановке $\mathrm{AR}(1)$-последовательностей с экспоненциальной инновацией.

В настоящей работе мы применяем, в основном, мартингальную технику, которая уже использовалась в [14], [15] и [17] для анализа распределения $\tau_{a}$ процессов авторегрессионного типа и, в частности, для процесса Орнштейна-У ленбека (т.е. процесса авторегрессии с непрерывным временем). Обзор некоторых результатов, полученных в этом направлении, можно найти в [18]. Из последних работ на эту тему укажем [7] и [8], в которых изучались процессы авторегрессионного типа с инновацией, имеющей распределение в виде смеси экспоненциальных функций.

2. Случай $|\lambda|<1$. Следующий результат содержит аналоги импликаций (2), (3) и (4).

Теорема 1. 1) Ecлu $|\lambda| \leqslant 1, b<0<a$, mo

$$
\mathbf{P}\left\{b(1-\lambda) \leqslant \eta_{1} \leqslant a(1-\lambda)\right\}<1 \Longrightarrow \tau_{a, b} \in \mathrm{Cr} .
$$

2) Eсли $\lambda \in(-1,0), a>0$ и для некоторого $\delta>0$

$$
\mathbf{E}\left|\eta_{1}\right|^{\delta}<\infty
$$

mo

$$
\mathbf{P}\left\{\eta_{1}+\lambda \eta_{2} \leqslant a\left(1-\lambda^{2}\right)\right\}<1 \Longrightarrow \tau_{a} \in \mathrm{Cr} .
$$

3) Eсли $\lambda \in(-1,0), a>0 u$

$$
E \ln \left(1+\left|\eta_{1}\right|\right)<\infty
$$

mo

$$
\mathbf{P}\left\{\eta_{1}+\lambda \eta_{2} \leqslant a\left(1-\lambda^{2}\right)\right\}<1 \Longrightarrow \mathbf{E}_{x} \tau_{a}<\infty .
$$

Д ок аз ат е ль с т в о. 1) В случае $\lambda \in[-1,0]$ при любом $n>1$ имеем

$$
\begin{aligned}
\mathbf{P}\left\{\tau_{a, b}>n\right\} & =\mathbf{P}\left\{\tau_{a, b}>n-1, X_{n-1} \in[b, a], X_{n} \in[b, a], \eta_{n}=X_{n}-\lambda X_{n-1}\right\} \\
& \leqslant \mathbf{P}\left\{\tau_{a, b}>n-1, \eta_{n} \in[b(1-\lambda), a(1-\lambda)]\right\} \\
& =\mathbf{P}\left\{\tau_{a, b}>n-1\right\} \mathbf{P}\left\{b(1-\lambda) \leqslant \eta_{n} \leqslant a(1-\lambda)\right\}
\end{aligned}
$$


где последнее равенство выполнено ввиду независимости $X_{k}$ и $\eta_{n}$ при $k<n$. По индукции

$$
\mathbf{P}\left\{\tau_{a, b}>n\right\} \leqslant\left[\mathbf{P}\left\{b(1-\lambda) \leqslant \eta_{n} \leqslant a(1-\lambda)\right\}\right]^{n},
$$

что влечет соотношение $\tau_{a, b} \in \mathrm{Cr}$ ввиду предположения $\mathbf{P}\left\{b(1-\lambda) \leqslant \eta_{n} \leqslant\right.$ $a(1-\lambda)\}<1$.

В случае $\lambda \in(0,1)$ мы можем использовать импликацию (4). Так как $\tau_{a, b} \leqslant \tau_{a}$, то в силу $(4)$

$$
\mathbf{P}\left\{\eta_{1} \leqslant a(1-\lambda)\right\}<1 \Longrightarrow \tau_{a, b} \in \mathrm{Cr} .
$$

По этой же причине (и ввиду симметрии обозначений) получаем

$$
\mathbf{P}\left\{\eta_{1} \geqslant b(1-\lambda)\right\}<1 \Longrightarrow \tau_{a, b} \in \mathrm{Cr} .
$$

Теперь остается заметить, что

$\mathbf{P}\left\{b(1-\lambda) \leqslant \eta_{1} \leqslant a(1-\lambda)\right\}<1 \Longleftrightarrow \mathbf{P}\left\{b(1-\lambda) \leqslant \eta_{1}\right\} \mathbf{P}\left\{\eta_{1} \leqslant a(1-\lambda)\right\}<1$.

Случай $\lambda=1$ отмечен выше в (2).

Для доказательства утверждений 2) и 3) заметим, что из (1) следует, что

$$
X_{2 k}=\lambda^{2} X_{2(k-1)}+\eta_{2 k}+\lambda \eta_{2 k-1}, \quad k=1,2, \ldots .
$$

Последовательность $\eta_{2 k}+\lambda \eta_{2 k-1}, k=1,2, \ldots$, является инновацией для последовательности $X_{2 k}, k=1,2, \ldots$. Поскольку время первого выхода $\widetilde{\tau}_{a}=\inf \left\{k \geqslant 0: X_{2 k}>a\right\}$ не меньше $\tau_{a} / 2$, то, применяя (4) к $\tilde{\tau}_{a}$, получаем (8). По таким же соображениям импликация (10) выполнена в силу теоремы 3 из [19]. Теорема 1 доказана.

Далее в этом пункте мы предполагаем, что $\lambda \neq 0,|\lambda|<1$ и выполнено следующее условие:

$$
\psi(u):=\ln \mathbf{E} e^{u \eta_{1}}<\infty \quad \text { для всех } \quad u \in(-\infty, \infty),
$$

которое, конечно, гарантирует выполнение условия (9).

Положим

$$
Q(y)=\frac{1}{\left|\ln \lambda^{2}\right|} \int_{0}^{\infty}\left[\left(e^{u y}-e^{u x}\right) e^{-\phi(u)}+\left(e^{-u y}-e^{-u x}\right) e^{-\phi(-u)}\right] \frac{d u}{u},
$$

где

$$
\phi(u)=\sum_{k=0}^{\infty} \psi\left(\lambda^{k} u\right)=\psi(u)+\phi(\lambda u), \quad u \in(-\infty, \infty) .
$$

Теорема 2. Пусть $|\lambda|<1, \lambda \neq 0, b<0<a$, выполнено (12) $u$

$$
\mathbf{P}\left\{b(1-\lambda) \leqslant \eta_{1}\right\}<1 \quad u \quad \mathbf{P}\left\{\eta_{1} \leqslant a(1-\lambda)\right\}<1 .
$$

Tогда

$$
\mathbf{E}_{x} \tau_{a, b}=\mathbf{E}_{x} Q\left(X_{\tau_{a, b}}\right)<\infty
$$


Д о к а з а т е л ь с т в о. Мы приводим здесь только набросок доказательства, поскольку оно близко к доказательству теоремы 3 из [19].

Сначала мы показываем, что процесс $Q\left(X_{n}\right)-n$ является мартингалом по отношению к естественной фильтрации $\mathscr{F}_{n}=\sigma\left\{X_{0}, X_{1}, \ldots, X_{n}\right\}$. Для случая $\lambda \in(0,1)$ этот факт следует из соотношения

$$
\begin{aligned}
Q\left(X_{n}\right)-n= & \frac{1}{2}\left[\frac{1}{|\ln \lambda|} \int_{0}^{\infty}\left(e^{u X_{n}}-e^{u x}\right) e^{-\phi(u)} \frac{d u}{u}-n\right] \\
& +\frac{1}{2}\left[\frac{1}{|\ln \lambda|} \int_{0}^{\infty}\left(e^{-u X_{n}}-e^{-u x}\right) e^{-\phi(-u)} \frac{d u}{u}-n\right],
\end{aligned}
$$

где процессы в квадратных скобках являются мартингалами (см. [19, утверждение 2 и теорема 2]).

Для случая $\lambda \in(-1,0)$ мы замечаем, что по лемме 1 из [19] интеграл в определении функции $Q(y)$ конечен при выполнении условий (12) и (15).

В силу (1), (12) и теоремы Фубини

$$
\begin{aligned}
& \mathrm{E}\left(Q\left(X_{n}\right)-n \mid \mathscr{F}_{n-1}\right) \\
& =\frac{1}{\left|\ln \lambda^{2}\right|} \int_{0}^{\infty}\left[\left(e^{u \lambda X_{n-1}+\psi(u)}-e^{u x}\right) e^{-\phi(u)}\right. \\
& \left.+\left(e^{-u \lambda X_{n-1}+\psi(-u)}-e^{-u x}\right) e^{-\phi(-u)}\right] \frac{d u}{u}-n \\
& =\frac{1}{\left|\ln \lambda^{2}\right|} \int_{0}^{\infty}\left[\left(e^{u \lambda X_{n-1}}-e^{\lambda u x}\right) e^{-\phi(\lambda u)}\right. \\
& \left.+\left(e^{-u \lambda X_{n-1}}-e^{-\lambda u x}\right) e^{-\phi(-\lambda u)}\right] \frac{d u}{u}-n \\
& +\int_{0}^{\infty} \frac{1}{\left|\ln \lambda^{2}\right|}\left[e^{u \lambda x-\phi(\lambda u)}+e^{-u \lambda x-\phi(-\lambda u)}-e^{u x-\phi(u)}-e^{-u x-\phi(-u)}\right] \frac{d u}{u} \\
& =Q\left(X_{n-1}\right)-n \\
& +\int_{0}^{\infty} \frac{1}{\left|\ln \lambda^{2}\right|}\left[e^{u \lambda x-\phi(\lambda u)}+e^{-u \lambda x-\phi(-\lambda u)}-e^{u x-\phi(u)}-e^{-u x-\phi(-u)}\right] \frac{d u}{u} .
\end{aligned}
$$

Последний интеграл равен 1 , так как его можно переписать в форме известного интеграла Фруллани (см. $[9$, п. 12.16]). Это означает, что процесс $Q\left(X_{n}\right)-n$ является мартингалом и, следовательно, по теореме об остановке мартингалов для любого $n=1,2, \ldots$

$$
\mathbf{E}_{x} \tau_{a, b}=\lim _{n \rightarrow \infty} \mathbf{E}_{x} Q\left(X_{\min \left(\tau_{a, b}, n\right)}\right) .
$$

Заключительный этап доказательства состоит в обосновании равенства

$$
\lim _{n \rightarrow \infty} \mathbf{E}_{x} Q\left(X_{\min \left(\tau_{a, b}, n\right)}\right)=\mathbf{E}_{x} Q\left(X_{\tau_{a, b}}\right),
$$

которое проводится аналогично доказательству сходного факта в [19]. 
Следствие 1. Пусть $|\lambda|<1, \lambda \neq 0, a>0, \eta_{1} \stackrel{d}{=}-\eta_{1}$ (символ $\stackrel{d}{=}$ означает равенство по распределению) $и$

$$
\mathbf{P}\left\{\eta_{1} \leqslant a(1-\lambda)\right\}<1 .
$$

Тогда

$$
\begin{aligned}
\mathbf{E}_{x} \tau_{a,-a} & =\frac{1}{|\ln | \lambda||} \int_{0}^{\infty}\left(\mathbf{E}_{x} \operatorname{ch}\left(u X_{\tau_{a,-a}}\right)-\operatorname{ch}(u x)\right) e^{-\phi(u)} \frac{d u}{u} \\
& \geqslant \frac{1}{|\ln | \lambda||} \int_{0}^{\infty}(\operatorname{ch}(u a)-\operatorname{ch}(u x)) e^{-\phi(u)} \frac{d u}{u} .
\end{aligned}
$$

Д о к а з а т е л ь с т в о. Для обоснования (16) нужно только заметить, что $\phi(u)=\phi(-u)$, и затем упростить выражение (13). Неравенство (17) справедливо в силу оценки $\operatorname{ch}\left(u X_{\tau_{a,-a}}\right) \geqslant \operatorname{ch}(u a)$.

Рассмотрим теперь случай гауссовской инновации $\eta_{n} \sim N\left(0, \varepsilon^{2}\right)$. В этом случае прямыми вычислениями легко получить, что

$$
\phi(u)=\frac{u^{2} \varepsilon^{2}}{2\left(1-\lambda^{2}\right)}
$$

Задача нахождения асимптотики $\mathbf{E}_{x} \tau_{a,-a}$ при $\varepsilon \rightarrow 0$ для гауссовского случая изучалась в [10], где, в частности, было показано, что при $|x|<1$

$$
\limsup _{\varepsilon \rightarrow 0} \varepsilon^{2} \ln \mathbf{E}_{x} \tau_{1,-1} \leqslant \frac{1-\lambda^{2}}{2}
$$

(см. теорему 2 в исправленной версии работы [10] или резюме доклада [21]). С помощью следствия 1 теперь мы получаем

Следствие 2. Пусть $|\lambda|<1, \lambda \neq 0,|x|<1, \eta_{1} \sim N\left(0, \varepsilon^{2}\right)$. Тогда

$$
\lim _{\varepsilon \rightarrow 0} \varepsilon^{2} \ln \mathbf{E}_{x} \tau_{1,-1}=\frac{1-\lambda^{2}}{2} .
$$

Д о к а з а т е л ь с т в о. В силу следствия 1

$$
\begin{aligned}
\mathbf{E}_{x} \tau_{1,-1} & \geqslant \frac{1}{|\ln | \lambda||} \int_{0}^{\infty}(\operatorname{ch} u-\operatorname{ch} x) \exp \left\{-\frac{u^{2} \varepsilon^{2}}{2\left(1-\lambda^{2}\right)}\right\} \frac{d u}{u} \\
& =\frac{1}{|\ln | \lambda||}\left(F\left(\frac{\sqrt{1-\lambda^{2}}}{\varepsilon}\right)-F\left(\frac{\sqrt{1-\lambda^{2}}}{\varepsilon}|x|\right)\right),
\end{aligned}
$$

где (например, с использованием программы Mathematica ${ }^{\circledR}$ )

$$
\begin{aligned}
F(x) & =\int_{0}^{\infty}(\operatorname{ch} u-1) e^{-u^{2} / 2} \frac{d u}{u}=\int_{0}^{x} \int_{0}^{\infty} \operatorname{sh}(u z) e^{-u^{2} / 2} d u d z \\
& =\sqrt{\frac{\pi}{2}} \int_{0}^{x} e^{z^{2} / 2} \operatorname{Erf}\left[\frac{z}{\sqrt{2}}\right] d z
\end{aligned}
$$


Так как $\operatorname{Erf}[z / \sqrt{2}] \rightarrow 1$ при $z \rightarrow \infty$, то (например, с использованием правила Лопиталя) получаем

$$
F(x) \sim \sqrt{\frac{\pi}{2}} \int_{0}^{x} e^{z^{2} / 2} d z \sim \sqrt{\frac{\pi}{2 x}} e^{x^{2} / 2} \quad \text { при } \quad x \rightarrow \infty .
$$

Последнее соотношение вместе с (20) и (18) влечет (19). Следствие 2 доказано.

Используя свойство самоподобия гауссовского распределения, можно легко показать, что математическое ожидание $\mathbf{E}_{x} \tau_{1,-1}$ при $\varepsilon=1 / a$ совпадает с $\mathbf{E}_{x a} \tau_{a,-a}$ при $\varepsilon=1$.

Таблицы 1 и 2 содержат результаты моделирования методом МонтеКарло для $\ln \mathbf{E}_{0} \tau_{a,-a}$ и соответствующие логарифмы нижней оценки $F\left(a \sqrt{1-\lambda^{2}}\right) /|\ln | \lambda \|($ см. (20)) при $\lambda=0.75$ и 0.9 для случая гауссовской инновации $\eta_{i} \sim N(0,1)$.

Для сравнения мы также включили логарифмы функции $F((a+$ $\left.0.5826) \sqrt{1-\lambda^{2}}\right) /|\ln | \lambda||$, которую мы называем скорректированной аппроксимацией для $\mathbf{E}_{0} \tau_{a,-a}$. Эта аппроксимация основана на предположении о том, что при относительно больших $a$ и $\lambda$, близких к 1 , математическое ожидание перескока $\mathrm{AR}(1)$-последовательности $X_{n}$ близко к константе $C=0.5826 \ldots$, которая является пределом математического ожидания перескока $X_{\tau_{a}}-a$ для гауссовского случайного блуждания $\sum_{k=1}^{n} \eta_{k}$ при $a \rightarrow \infty$ (см. [23]).

Таблица 1. $\lambda=0.75,\left(1-\lambda^{2}\right) / 2=0.219$

\begin{tabular}{|c|c|c|c|c|c|}
\hline$a$ & 4 & 5 & 6 & 7 & 8 \\
\hline $\ln ($ нижняя граница $) / a^{2}$ & 0.257 & 0.234 & 0.224 & 0.219 & 0.217 \\
\hline $\ln$ (скорректированная аппр.) $/ a^{2}$ & 0.317 & 0.283 & 0.265 & 0.255 & 0.248 \\
\hline $\ln \left(\right.$ Монте-Карло) $/ a^{2}$ & 0.325 & 0.288 & 0.268 & 0.258 & 0.249 \\
\hline
\end{tabular}

Таблица 2. $\lambda=0.9,\left(1-\lambda^{2}\right) / 2=0.095$

\begin{tabular}{|c|c|c|c|c|}
\hline$a$ & 4 & 6 & 8 & 10 \\
\hline $\ln ($ нижняя граница)/a & 0.203 & 0.140 & 0.116 & 0.106 \\
\hline $\ln$ (скорректированная аппр.)/a & 0.234 & 0.157 & 0.129 & 0.116 \\
\hline $\ln$ (Монте-Карло) $/ a^{2}$ & 0.236 & 0.158 & 0.130 & 0.117 \\
\hline
\end{tabular}

Таблицы 1 и 2 свидетельствуют о том, что скорректированная аппроксимация дает намного лучшее приближение к результатам моделирования по сравнению с нижней оценкой и тем более по сравнению с предельным значением $\left(1-\lambda^{2}\right) / 2$ (в логарифмической шкале). 


\section{3. Случай инновации с экспоненциальным распределением.} Мы будем использовать здесь стандартные обозначения из теории $q$-рядов (см. [1]) и, в частности, $q$-символ Похгаммера:

$$
\prod_{j=1}^{m}\left(1-\rho \lambda^{j-1}\right)=:(\rho ; \lambda)_{m}, \quad(\rho ; \lambda)_{0}=1 .
$$

В частности, используя это обозначение, имеем

$$
(\lambda ; \lambda)_{m}=\prod_{j=1}^{m}\left(1-\lambda^{j}\right) .
$$

Положим

$$
\begin{aligned}
N_{\rho}(x) & =\sum_{k=0}^{\infty} \frac{(\lambda x)^{k}(\rho ; \lambda)_{k}}{k !}, \quad 0<\rho<1 ; \\
H(x) & =\sum_{k=1}^{\infty} \frac{x^{k}(\lambda ; \lambda)_{k-1}}{k !}
\end{aligned}
$$

Теорема 3. Пусть $0<\lambda<1, x \in[0, a], a>0, \mathbf{P}\left\{\eta_{1}>y\right\}=e^{-y}$, $y>0$. Тогда

1) для любого $\rho \in(0,1)$ выполнено равенство

$$
\mathbf{E}_{x} \rho^{\tau_{a}}=\frac{\rho N_{\rho}(x)}{N_{\rho}(a / \lambda)}
$$

2) выполнено равенство

$$
\mathbf{E}_{x} \tau_{a}=H(a)-H(\lambda x)+1 .
$$

Д о к а $з$ а т е л ь с т в о. Покажем сначала, что процесс $\rho^{n} N_{\rho}\left(X_{n}\right)$ является мартингалом. Нетрудно проверить, что для этого функция $N_{\rho}(x)$ должна удовлетворять уравнению

$$
\rho \mathbf{E} N_{\rho}\left(\lambda x+\eta_{1}\right)=N_{\rho}(x)
$$

(см. также [19, утверждение 1]).

Так как $\mathbf{E} N_{\rho}\left(\lambda x+\eta_{1}\right)=\int_{0}^{\infty} N_{\rho}(\lambda x+y) e^{-y} d y$, то после замены $\lambda x+$ $y=z$ получаем

$$
\rho \int_{\lambda x}^{\infty} N_{\rho}(z) e^{-z} d z=e^{-\lambda x} N_{\rho}(x) .
$$

После дифференцирования по $x$ левой и правой части и затем некоторых упрощений получим

$$
N_{\rho}^{\prime}(x)=\lambda N_{\rho}(x)-\lambda \rho N_{\rho}(\lambda x) .
$$


Будем решать это уравнение методом степенных рядов, предполагая, что $N_{\rho}(x)=\sum_{k=0}^{\infty} c_{k} x^{k}, c_{0}=1$. Тогда, подставляя этот ряд в (26), имеем

$$
\sum_{k=0}^{\infty} c_{k+1}(k+1) x^{k}=\sum_{k=0}^{\infty} c_{k} \lambda\left(1-\rho \lambda^{k}\right) x^{k} .
$$

Приравнивая коэффициенты в левой и правой частях, получаем $c_{k+1}(k+1)=c_{k} \lambda\left(1-\rho \lambda^{k}\right)$. Отсюда вытекает, что $c_{k}=\lambda^{k}(\rho ; \lambda)_{k} / k !$ и, следовательно, функция $N_{\rho}(x)$ действительно имеет представление $(22)$.

Применяя теорему об остановке мартингалов, получим, что при любом $n=1,2, \ldots$

$$
\mathbf{E}_{x}\left(\rho^{\tau_{a}} I\left\{\tau_{a} \leqslant n\right\} N_{\rho}\left(X_{\tau_{a}}\right)\right)+\mathbf{E}_{x}\left(\rho^{n} I\left\{\tau_{a}>n\right\} N_{\rho}\left(X_{n}\right)\right)=N_{\rho}(x) .
$$

Так как на множестве $\left\{\tau_{a}>n\right\}$, очевидно, выполнена оценка $N_{\rho}\left(X_{n}\right) \leqslant$ $N_{\rho}(a)$, то, переходя к пределу при $n \rightarrow \infty$, получим

$$
\mathbf{E}_{x}\left(\rho^{\tau_{a}} I\left\{\tau_{a}<\infty\right\} N_{\rho}\left(X_{\tau_{a}}\right)\right)=N_{\rho}(x) .
$$

Из (27), в частности, следует, что $\mathbf{P}\left\{\tau_{a}<\infty\right\}=1$, поскольку имеется монотонная сходимость $N_{\rho}(x) \rightarrow 1$ при $\rho \rightarrow 1$ и любом $x \geqslant 0$.

Теперь мы воспользуемся известным (по крайней мере, в фольклоре) фактом о том, что

$$
X_{\tau_{a}}-a \stackrel{d}{=} \eta_{1} ; \quad X_{\tau_{a}} \text { и } \tau_{a} \text { независимы. }
$$

Этот факт указан для случайных блужданий (т.е. при $\lambda=1$ ), например, в [4]; см. также обобщение этого факта в теореме 4 ниже.

Ввиду указанного факта и (27), имеем

$$
\mathbf{E}_{x} \rho^{\tau_{a}}=\frac{N_{\rho}(x)}{\mathbf{E}_{x} N_{\rho}\left(X_{\tau_{a}}\right)}
$$

где в силу (28)

$$
\mathrm{E}_{x} N_{\rho}\left(X_{\tau_{a}}\right)=\mathbf{E}_{x} N_{\rho}\left(a+\eta_{1}\right)=\frac{N_{\rho}(a / \lambda)}{\rho} .
$$

Таким образом, (23) доказано.

Для доказательства второй части теоремы 3 мы рассмотрим асимптотическое разложение математического ожидания $\mathbf{E}_{x} \rho^{\tau_{a}}$ при $\rho \rightarrow 1$. Заметим, что $\left(1-\mathbf{E}_{x} \rho^{\tau_{a}}\right) /(1-\rho) \rightarrow \mathbf{E}_{x} \tau_{a}$. С другой стороны, при $k \geqslant 1$ имеем $(\rho ; \lambda)_{k} /(1-\rho) \rightarrow(\lambda ; \lambda)_{k-1}$ и, следовательно,

$$
N_{\rho}(x)=1+(1-\rho)\left(\lambda x+\sum_{k=2}^{\infty} \frac{(\lambda x)^{k}(\lambda ; \lambda)_{k-1}}{k !}\right)+o(1-\rho) .
$$


Отсюда вытекает, что

$$
\begin{aligned}
\frac{1-\mathbf{E}_{x} \rho^{\tau_{a}}}{1-\rho}= & \frac{\sum_{k=0}^{\infty} a^{k}(\rho ; \lambda)_{k} / k !-\rho \sum_{k=0}^{\infty}(x \lambda)^{k}(\rho ; \lambda)_{k} / k !}{(1-\rho) \sum_{k=0}^{\infty} a^{k}(\rho, \lambda)_{k} / k !} \\
= & \left(1+\sum_{k=1}^{\infty}\left(\frac{a^{k}(\lambda ; \lambda)_{k-1}}{k !}-\frac{(x \lambda)^{k}(\lambda ; \lambda)_{k-1}}{k !}\right)+o(1-\rho)\right) \\
& \times(1+o(1))^{-1} \longrightarrow H(a)-H(\lambda x)+1=\mathbf{E}_{x} \tau_{a} .
\end{aligned}
$$

Теорема 3 доказана.

Следующая теорема обобщает использованный выше результат (28) на случай нелинейных границ и $\lambda \in(0,1]$.

Теорема 4. Пусть $\lambda \in(0,1], \mathbf{P}\left\{\eta_{1}>y\right\}=e^{-y}, y>0, u$

$$
\tau_{f}=\inf \left\{n \geqslant 0: X_{n}>f(n)\right\}, \quad f(0)>x, \quad \Upsilon_{f}=X_{\tau_{f}}-f\left(\tau_{f}\right),
$$

где $f(n)$ - неслучайная Функиия. Тогда для любого $n>0$ и $y \geqslant 0$

$$
\mathbf{P}\left\{\Upsilon_{f}>y, \tau_{f} \leqslant n\right\}=e^{-y} \mathbf{P}\left\{\tau_{f} \leqslant n\right\} .
$$

Д о к а з а т ел ь с т в о. В силу марковского свойства AR(1)-последовательности

$$
\begin{aligned}
\mathbf{P} & \left\{\Upsilon_{f}>y, \tau_{f} \leqslant n\right\}=\sum_{k=1}^{n} \mathbf{P}\left\{X_{k}>f(k)+y \mid \tau=k\right\} \mathbf{P}\left\{\tau_{f}=k\right\} \\
& =\sum_{k=1}^{n} \mathbf{P}\left\{X_{k}>f(k)+y \mid X_{k-1} \leqslant f(k-1), X_{k}>f(k)\right\} \mathbf{P}\left\{\tau_{f}=k\right\} \\
& =\sum_{k=1}^{n} \frac{\mathbf{P}\left\{\eta_{k}>f(k)-\lambda X_{k-1}+y, X_{k-1} \leqslant f(k-1)\right\}}{\mathbf{P}\left\{\eta_{k}>f(k)-\lambda X_{k-1}, X_{k-1} \leqslant f(k-1)\right\}} \mathbf{P}\left\{\tau_{f}=k\right\} .
\end{aligned}
$$

Так как при любом $y \geqslant 0$

$$
\begin{aligned}
\mathbf{P} & \left\{\eta_{k}>f(k)-\lambda X_{k-1}+y, X_{k-1} \leqslant f(k-1)\right\} \\
& =\int_{-\infty}^{f(k-1)} \mathbf{P}\left\{\eta_{k}>f(k)-\lambda z+y\right\} \mathbf{P}\left\{X_{k-1} \in d z\right\} \\
& =e^{-f(k)-y} \int_{-\infty}^{f(k-1)} e^{\lambda z} \mathbf{P}\left\{X_{k-1} \in d z\right\}
\end{aligned}
$$

(мы воспользовались независимостью случайных величин $X_{k-1}$ и $\eta_{k}$ ), то (29) равно $\sum_{k=1}^{n} e^{-y} \mathbf{P}\left\{\tau_{f}=k\right\}=e^{-y} \mathbf{P}\left\{\tau_{f} \leqslant n\right\}$. Теорема 4 доказана.

\section{4. Одна задача об оптимальной остановке для $\mathrm{AR}(1)$-по-} следовательностей. Здесь мы обсуждаем классическую задачу об оптимальной остановке, которая заключается в нахождении функции (цены)

$$
V_{\mathscr{M}}(x)=\sup _{\tau \in \mathscr{M}} \mathbf{E}_{x}\left[\rho^{\tau} g\left(X_{\tau}\right) I\{\tau<\infty\}\right]
$$


где $\rho \in(0,1), g(x)$ - неотрицательная функция выплат, $\mathscr{M}$ - заданный класс моментов остановки по отношению к естественной фильтрации $\mathscr{F}_{n}=\sigma\left\{X_{0}, X_{1}, \ldots, X_{n}\right\}, X_{n}-\mathrm{AR}(1)$-последовательность. В качестве $\mathscr{M}$ обычно рассматриваются либо $\overline{\mathscr{M}}$ - класс всех моментов остановки, либо класс ограниченных моментов остановки

$$
\mathscr{M}_{T}=\{\tau \in \overline{\mathscr{M}}: \tau \leqslant T\} .
$$

Изложение общей теории задач об оптимальной остановке с большим количеством примеров имеется в [6], см. также [22], [5], [20]. Известно, что $V \overline{\mathscr{M}}(x)$ является решением уравнения Вальда-Беллмана, которое в рассматриваемом нами случае имеет следующий вид:

$$
V_{\overline{\mathscr{M}}}(x)=\max \left\{g(x), \rho \mathbf{E}_{x} V_{\overline{\mathscr{M}}}\left(\lambda x+\eta_{1}\right)\right\} .
$$

Насколько мы знаем, явное решение этого уравнение не было известно ни для каких нетривиальных примеров $\mathrm{AR}(1)$-последовательностей с $\lambda \in(0,1)$ (случай $g(x)=$ const не представляет интереса).

Далее мы предполагаем, что $\mathbf{P}\left\{\eta_{1}>y\right\}=e^{-y}, y>0$.

Используя лемму Фату, получаем

$$
\begin{aligned}
V_{\overline{\mathscr{M}}}(x) & \leqslant \sup _{\tau \in \overline{\mathscr{M}}} \sup _{a>0} \mathbf{E}_{x}\left[\rho^{\min \left(\tau_{a}, \tau\right)} N_{\rho}\left(X_{\min \left(\tau_{a}, \tau\right)}\right) \frac{g\left(X_{\min \left(\tau_{a}, \tau\right)}\right)}{N_{\rho}\left(X_{\min \left(\tau_{a}, \tau\right)}\right)}\right] \\
& \leqslant \sup _{\tau \in \overline{\mathscr{M}}} \sup _{a>0} \mathbf{E}_{x}\left[\rho^{\min \left(\tau_{a}, \tau\right)} N_{\rho}\left(X_{\min \left(\tau_{a}, \tau\right)}\right)\right] A
\end{aligned}
$$

где $A=\sup _{y>0}\left(g(y) / N_{\rho}(y)\right)$ и по мартингальному свойству процесса $\rho^{n} N_{\rho}\left(X_{n}\right)$ (см. доказательство теоремы 3) $\mathbf{E}_{x}\left[\rho^{\min \left(\tau_{a}, \tau\right)} N_{\rho}\left(X_{\min \left(\tau_{a}, \tau\right)}\right)\right]=$ $N_{\rho}(x)$. Поскольку из $(22)$ следует, что для функции $N_{\rho}(y)$ выполнены неравенства $(\rho ; \lambda)_{\infty} \exp \{\lambda y\} \leqslant N_{\rho}(y) \leqslant \exp \{\lambda y\}$, то константа $A$ конечна для непрерывных функций выплат $g(x)$, удовлетворяющих условию

$$
\limsup _{x \rightarrow \infty} g(x) \exp \{-\lambda x\}<\infty .
$$

Чтобы получить нижнюю оценку для $V_{\bar{M}}(x)$, заметим, что

$$
V_{\overline{\mathscr{M}}}(x) \geqslant \sup _{a>0} \mathbf{E}_{x}\left[\rho^{\tau_{a}} g\left(X_{\tau_{a}}\right)\right]
$$

где, с использованием (28) и теоремы 3 , имеем

$$
\mathbf{E}_{x}\left[\rho^{\tau_{a}} g\left(X_{\tau_{a}}\right)\right]=\mathbf{E}_{x} \rho^{\tau_{a}} \mathbf{E}_{x} g\left(X_{\tau_{a}}\right)=\rho N_{\rho}(x) \frac{\mathbf{E} g\left(a+\eta_{1}\right)}{N_{\rho}(a / \lambda)}
$$

Так как всегда $V_{\bar{M}}(x) \geqslant g(x)$, то из $(32)$ и $(33)$ получается следующая нижняя оценка:

$$
V_{\overline{\mathscr{M}}}(x) \geqslant G(x):=\max \left\{g(x), \rho N_{\rho}(x) \sup _{a>0} \frac{\mathbf{E} g\left(a+\eta_{1}\right)}{N_{\rho}(a / \lambda)}\right\} .
$$


Этот результат наводит на предположение, что в классе $\overline{\mathscr{M}}$ оптимальным моментом остановки может быть (при некоторых дополнительных ограничениях на функцию выплат $g(x))$ время пересечения уровня $a^{*}$, на котором достигается максимум функции $B(a)=\mathbf{E} g\left(a+\eta_{1}\right) / N_{\rho}(a / \lambda)$. Один из возможных путей проверки этого предположения состоит в доказательстве того, что нижняя оценка является единственным (в подходящем классе функций) решением уравнения Вальда-Беллмана (30). Доказательство нижеследующего результата основано на другом подходе, который заключается в проверке того, что нижняя оценка является также и верхней оценкой для функции цены (этот подход использовался, например, в [20] для случайных блужданий и процессов Леви).

Теорема 5. Пусть $\lambda \in(0,1), \mathbf{P}\left\{\eta_{1}>y\right\}=e^{-y}, y>0, u g(x)-$ непрерывная неубывающая функция выплат, $g(0)=0$. Предположим также, что функиия $B(a)=\mathbf{E} g\left(a+\eta_{1}\right) / N_{\rho}(a / \lambda)$ имеет единственный максимум в точке $a^{*}>0$. Тогда при $x \in\left[0, a^{*}\right)$ момент остановки

$$
\tau_{a^{*}}=\inf \left\{n \geqslant 0: X_{n}>a^{*}\right\}
$$

является оптимальньц в классе $\overline{\mathscr{M}}$ u

$$
V_{\overline{\mathscr{M}}}(x)=\rho N_{\rho}(x) \frac{\mathbf{E} g\left(a^{*}+\eta_{1}\right)}{N_{\rho}\left(a^{*} / \lambda\right)} .
$$

Д о к а з а т е л ь с т в о. Полученная выше нижняя оценка (34) в силу условия о неубывании и непрерывности функции $g(x)$ и других условий теоремы 5 имеет следуюший вид:

$$
V_{\bar{M}}(x) \geqslant G(x):=\rho N_{\rho}(x) \sup _{a>0} \frac{\mathbf{E} g\left(a+\eta_{1}\right)}{N_{\rho}(a / \lambda)}=\rho N_{\rho}(x) B\left(a^{*}\right) .
$$

Чтобы показать, что $V_{\overline{\mathscr{M}}}(x) \leqslant G(x)$, заметим сначала, что в силу неотрицательности $g(x)$

$$
V_{\overline{\mathscr{M}}}(x) \leqslant \sup _{T>0} V_{\mathscr{M}_{T}}(x) .
$$

Хорошо известно, что оптимальный момент остановки в классе $\mathscr{M}_{T}$ имеет следующий вид:

$$
\tau_{\mathrm{opt}}=\tau_{f}=\min \left\{n \leqslant T: g\left(X_{n}\right)=V_{\mathscr{M}_{T-n}}(x)\right\}=\min \left\{n \leqslant T: X_{n}>f_{n}\right\},
$$

где $f=f(n)$ - некоторая детерминированная граница и последнее равенство справедливо в силу условий неубывания и непрерывности функции $g(x)$ (и непрерывности распределения $X_{n}$ ).

Таким образом,

$$
V_{\mathscr{M}_{T}}(x)=\mathbf{E}_{x}\left[\rho^{\tau_{f}} g\left(X_{\tau_{f}}\right)\right]=\mathbf{E}_{x}\left[\rho^{\tau_{f}} \mathbf{E}_{x}\left(g\left(f\left(\tau_{f}\right)+\eta_{1}\right) \mid \tau_{f}\right)\right],
$$


где мы воспользовались доказанным выше (см. теорему 4) фактом о независимости $\tau_{f}$ и перескока $X_{\tau_{f}}-f\left(\tau_{f}\right) \stackrel{d}{=} \eta_{1}$. Так как процесс $\rho^{n} N_{\rho}\left(X_{n}\right)$ является мартингалом, то

$$
\mathbf{E}_{x} \rho^{\tau_{f}} N_{\rho}\left(X_{\tau_{f}}\right)=N_{\rho}(x)
$$

С другой стороны, опять же в силу независимости $\tau_{f}$ и перескока $X_{\tau_{f}}$ $f\left(\tau_{f}\right) \stackrel{d}{=} \eta_{1}$, имеем

$$
\mathbf{E}_{x} \rho^{\tau_{f}} N_{\rho}\left(X_{\tau_{f}}\right)=\mathbf{E}_{x} \rho^{\tau_{f}} \mathbf{E}_{x} N_{\rho}\left(X_{\tau_{f}}\right)
$$

Из последних двух соотношений и (35) следует, что

$$
\begin{aligned}
V_{\mathscr{M}_{T}}(x) & \leqslant \mathbf{E}_{x}\left[\rho^{\tau_{f}} \mathbf{E}_{x}\left(N_{\rho}\left(f\left(\tau_{f}\right)+\eta_{1}\right) \mid \tau_{f}\right) \sup _{\tau_{f}} \frac{\mathbf{E}_{x}\left(g\left(f\left(\tau_{f}\right)+\eta_{1}\right) \mid \tau_{f}\right)}{\mathbf{E}_{x}\left(N_{\rho}\left(f\left(\tau_{f}\right)+\eta_{1}\right) \mid \tau_{f}\right)}\right] \\
& \leqslant \mathbf{E}_{x}\left[\rho^{\tau_{f}} \mathbf{E}_{x}\left(N_{\rho}\left(f\left(\tau_{f}\right)+\eta_{1}\right) \mid \tau_{f}\right)\right] \sup _{a} \frac{\mathbf{E} g\left(a+\eta_{1}\right)}{\mathbf{E} N_{\rho}\left(a+\eta_{1}\right)} \\
& =N_{\rho}(x) \sup _{a} \frac{\mathbf{E} g\left(a+\eta_{1}\right)}{\mathbf{E} N_{\rho}\left(a+\eta_{1}\right)} .
\end{aligned}
$$

Чтобы завершить доказательство, остается заметить, что последний супремум равен $\rho B\left(a^{*}\right)$.

3 а м е ч а н и е. Доказательство теоремы 5 было бы проще, если бы было известно, что в классе $\overline{\mathscr{M}}$ оптимальный момент остановки является моментом выхода за уровень. Такого типа результаты известны для процессов Орнштейна-Уленбека с непрерывным временем, не имеющих положительных скачков (см., например, статью [3], в которой существенно использовалась техника и результаты статей [13] и [16]).

В заключение, автор выражает признательность Брите Рус и Фиме Клебанеру за стимулирующие обсуждения результатов работы [10], а также Нино Кордзахия, Юпапорн Ареепонг, Габриелу Митетели, Саованит Сакпарунгси и рецезенту за полезные замечания.

\section{СПИСОК ЛИТЕРАТУРЫ}

1. Andrews G.E., Askey R., Roy R. Special Functions. Cambridge: Cambridge Univ. Press, 1999, $664 \mathrm{c}$.

2. Areepong Y., Novikov A.A. EWMA control charts for changes in exponential distributions. - J. Quality Measurement and Anal. (to appear).

3. Baurdoux E.J. Examples of optimal stopping via measure transformation for processes with one-sided jumps. - Stochastics, 2007, v. 79, № 3-4, p. 303-307.

4. Боровков A. A. Вероятностные процессы в теории массового обслуживания. M.: Наука, 1972, 367 c.

5. Роббинс Г., Сигмунд Д., Чао И. Теория оптимальных правил остановки. М.: Наука, 1977, 167 с. 
6. Peskir G., Shiryaev A.N. Optimal Stopping and Free-Boundary Problems. Basel: Birkhäuser, 2006, $500 \mathrm{p}$.

7. Jacobsen M., Jensen A.T. Exit times for a class of piecewise exponential Markov processes with two-sided jumps. - Stochastic Process. Appl., 2007, v. 117, №9, p. 1330-1356.

8. Jacobsen $M$. Exit times for a class of autoregressive sequences and random walks. Working paper. Copenhagen: Dept. of Appl. Math. and Statist., Univ. of Copenhagen.

9. Джеффрис Г., Свирлс Б. Методы математической физики, т. 2. М.: Мир, 1969, $352 \mathrm{c}$

10. Клебанер Ф. К., Липцер Р. IШ. Большие уклонения для рекуррентных последовательностей с последействием. - Проблемы передачи информ., 1996, т. 32, № 4, c. 23-34; испр. версия: http://arxiv.org/PS_cache/math/pdf/0603/0603407v1.pdf.

11. Kyprianou A.E. Introductory Lectures on Fluctuations of Lévy Processes with Applications. Berlin: Springer-Verlag, 2006, 373 p.

12. Larralde $H$. A first passage time distribution for a discrete version of the OrnsteinUhlenbeck process. - J. Phys. A, 2004, v. 37, № 12, p. 3759-3767.

13. Lerche H. R., Urusov M. Optimal stopping via measure transformation: the BeibelLerche approach. - Stochastics, 2007, v. 79, № 3-4, p. 275-291.

14. Новиков $A$. A. О моменте первого выхода процесса авторегрессии за уровень и одно применение в задаче «разладки». - Теория вероятн. и ее примен., 1990, т. 35 , в. 2 , с. $282-292$.

15. Новиков А.А., Эргашев Б.А. Предельные теоремы для момента достижения уровня процессом авторегрессии. - Тр. МИАН, 1993, т. 202, с. 209-233.

16. Новиков A. A. Мартингалы и моменты первого выхода для процесса ОрнштейнаУленбека со скачками. - Теория вероятн. и ее примен., 2003, т. 48, в. 2, c. 340-358.

17. Novikov A. A., Melchers R.E., Shinjikashvili E., Kordzakhia N. First passage time of filtered Poisson process with exponential shape function. - Probab. Engrg. Mech., 2005 , v. 20, № 1, p. 33-44.

18. Novikov A. A. Lévy-driven Ornstein-Uhlenbeck processes: survey of results on first passage times. - Lecture notes at the conference «Stochastic Calculus with Jumps». Univ. of Angers, 2006; http://www.im.pwr.wroc.pl/ jumps/ln/novikov.pdf

19. Novikov A.A., Kordzakhia N. Martingales and first passage times of AR(1) processes. - Stochastics, 2008, v. 80, № 2-3, p. 197-210.

20. Novikov A.A., Shiryaev A.N. On a solution of the optimal stopping problem for processes with independent increments. - Stochastics, 2007, v. 79, p. 393-406.

21. Ruths B. Exit times for past dependent systems. - Extended abstracts of RussianScandinavian Symposium on Probability Theory and Applied Probability, Petrozavodsk, 2006, p. 72.

22. Ширяев A.Н. Статистический последовательный анализ: оптимальные правила остановки. М.: Наука, 1976, 272 с.

23. Siegmund D. Sequential Analysis - Tests and Confidence Intervals. New York: Springer-Verlag, 1985, $272 \mathrm{p}$.

24. Stein C. A note on cumulative sums. - Ann. Math. Statist., 1946, v. 17, p. 498-499.

25. Vervaat $V$. On a stochastic difference equation and a representation of nonnegative infinitely divisible random variables. - Adv. Appl. Probab., 1979, v. 11, p. 750-783.

26. Wijsman R. A. Stopping times: termination, moments, distribution. - Handbook of Sequential Analysis. New York: Dekker, 1991, p. 67-119.

Поступила в редакцию 16.X.2007

Исправленный вариант 3.III. 2008 